\title{
Público/Privado versus Derecho/Negocio en Materia de Salud
}

\author{
Francisco Angora Mazuecos ${ }^{a}$
}

\begin{abstract}
a Médico de Atención Primaria, miembro de la Federación de Asociaciones para la Defensa de la Sanidad Pública y Gerente de Atención Primaria (SESCAM) en el Área de Salud de Ciudad Real.
\end{abstract}

Correspondencia: Francisco Angora Mazuecos. Avda. Pío XII s/n. 13002 - Ciudad Real. Correo electrónico: fangoram@sescam.jccm.es

Recibido el 16 de marzo de 2011.

Aceptado para su publicación el 17 de abril de 2011.

\section{RESUMEN}

La aceleración del gasto sanitario en el Servicio Nacional de Salud (SNS), postulada como insostenible hace 21 años en el "informe Abril"1 de 1991 y de nuevo en el "informe McKinsey \& Company" (M\&C) $)^{2}$ de 2009, se basó entre otros motivos en la mala gestión y el envejecimiento poblacional. Ambos contienen errores de bulto, y tal vez forzados, para abrir puertas a la progresiva privatización, que sólo han servido para encarecer, cual diezmo, los costes de la asistencia sanitaria allí donde se implantaron.

El Servicio de Salud de Castilla-La Mancha (SESCAM), como en general el SNS, demuestra altos grados de eficiencia año tras año, con mejores resultados, alta satisfacción de la población y mayor esperanza de vida que la media de los países de la Unión Europea y de la OCDE. Las medidas estrella propugnadas en ambos informes, copago y autogestión, se desestiman aquí por los fracasos cosechados en otros Países y algunas Comunidades Autónomas Españolas. Proponemos introducir reformas e innovaciones, pero dentro de los márgenes saludables de mejora de la eficiencia en gestión sanitaria.

Palabras clave. Servicios de Salud, Eficiencia.

\section{ABSTRACT}

Public/Private versus Rights/Business in Healthcare issue

The escalation of healthcare expenditure in the National Health Service (NHS), postulated as unsustainable 21 years ago in the 1991 April report ${ }^{1}$ and again in the 2009 McKinsey \& Company" (M\&c) report ${ }^{2}$, was based on bad management and the aging population, amongst other reasons. Both reports contain blunders, perhaps coerced, in order to lead the way to gradual privatisation, which has only served to increase the cost of healthcare where it was implemented.

The Castilla-La Mancha Healthcare Service (SESCAM) and the NHS in general, have shown a high level of efficiency year after year, with better results, a high level of population satisfaction and a longer mean life expectancy than that of other EU and OCDE countries.

The star measures advocated in both reports, co-payment and self-management, are rejected here due to their failure in other countries and in some Spanish autonomous communities.

We propose the implementation of reforms and innovations, but those that are within the healthy margins of improving the efficiency of healthcare management.

Key words. Health Services, Efficiency.

\section{PLANTEAMIENTO}

A finales de 2009 se publicó un informe de M\&C sobre el riesgo de sostenibilidad del sistema sanitario público español y la correspondiente necesidad de tomar medidas, por supuesto en línea con aquellas de las que se nutren empresas como la citada multinacional estadounidense, que entienden la sanidad ante todo como oportunidad de negocio, lógico por otra parte de entidades que invierten en lo mas rentable del mercado, sin más escrúpulos.

He creído de interesante actualidad revisar el artículo de la Federación de Asociaciones para la Defensa de la Sanidad Pública (FADSP) ${ }^{3}$ al respecto, escrito en enero de 2010, ya en plena crisis financiera. Ahora que los tentáculos de este tipo de macroempresas se acercan incluso a puertos 
antes poco apetecibles como es el caso de Castilla-La Mancha, donde el Gobierno Regional se decanta manifiestamente por mantener el Servicio de Salud Público. La aproximación la hacen precedida de toda la orquesta mediática que habla a ciudadanos, profesionales y políticos, cual canto de sirenas, de las ventajas de la privatización más o menos encubierta, a veces bajo fórmulas de colaboración público-privadas (CPP) para las prestaciones sanitarias, en función de una situación supuestamente crítica. El informe M\&C, a mi juicio, basa sus argumentos en errores, de bulto o forzados, del tipo que referimos a continuación.

\section{ARGUMENTOS NEOLIBERALES/ CONTRAARGUMENTOS}

- En contra de lo que enuncian, el SNS sí es globalmente eficiente, como lo es el SESCAM, consiguiendo buenos resultados, con un gasto sanitario total del $8,4 \%$ del PIB, inferior al de la media de los 15 de la UE que es del 9,6\%4 y en la media de la $U^{27}$, siendo significativo el "descuelgue" sanitario-financiero de las autonomías que se han desmarcado hacia la privatización, como es el caso de Madrid, Valencia, Cataluña, Galicia, Murcia y en parte Andalucía ${ }^{5}$.

- Los costes medios de los Servicios Sanitarios no dependen, como pretenden en su informe, sólo de los porcentajes de población mayor de 65 años, sino más bien de la incorporación incontrolada y a veces injustificada de novedades tecnológicas y farmacológicas que no aportan valor añadido, pero sí que logran introducirse y ser demandadas por ciudadanos y profesionales en función del correspondiente marketing, como también ocurre con la prescripción de marcas de fantasía en lugar de por principio activo y/o genéricos, a pesar de las recomendaciones de las agencias europea y española del medicamento y de las sociedades científicas del ramo.

- La supuesta aceleración del gasto, utilizada como insostenible per se en el informe Abril de julio del 1991, es evidente que no es tal, puesto que han pasado 20 años y el gasto sanitario de España sigue estando en la media de los países de la UE sobre el correspondiente PIB.

- La mayor presión asistencial y la frecuentación o número de visitas que el médico de Atención
Primaria (AP) recibe de cada paciente al año, y que supera en un $20 \%$ a la media europea, responde a la mayor esperanza de vida española y también a la gran carga burocrática que soporta la AP y que está actualmente en trámites de mejora en la mayoría de CCAA mediante diferentes innovaciones organizacionales sobre los temas de recetas, incapacidad temporal, certificaciones de todo tipo, justificantes, duplicidades de tareas y empoderamiento para la responsabilización e incremento de autonomía de los pacientes crónicos. Por otro lado, no está claro si en algunos países se consulta menos de lo que sería apropiado.

En conclusión, no existe evidencia de que el sistema sanitario español corra riesgos diferentes a los de cualquier sistema sanitario de Europa o del mundo, y sí que la hay de que aquí se consigue un elevado nivel de salud a bajo coste.

Ninguna Comunidad Autónoma está a salvo de los ataques neoliberalistas que buscan tajada a través de la mercantilización de la salud, y ya no solo por las vías clásicas de la tecnología y la industria farmacéutica, sino desembarcando en nuestros hasta ahora humildes puertos sanitarios con intención de gestionarlos, en aras de disminuir costes, mejorar en eficiencia y "hacer mejor las cosas"; falacias suficientemente contrastadas y fracasadas fuera ${ }^{6,4}$ y dentro de España, con el resultado del retroceso de diferentes indicadores allí donde se implantaron. Los beneficios de los "intermediarios", basados en el mercado, la competencia o la participación privada en la financiación y gestión de los Centros Sanitarios Públicos, condujeron por ejemplo a la quiebra del National Health Service (NHS) del Reino Unido, debido al incremento de los costes, que llega a duplicarse, y al endeudamiento que generan, que se multiplica sobre el basal público. Cuestión aparte es la provisión de productos de consumo que el sistema precisa adquirir de fuentes centralizadas buscando la máxima efectividad económica, como recomienda el propio Consejo Interterritorial.

\section{PROPUESTAS NEOLIBERALES/ CONTRAPROPUESTAS}

Entre las medidas que en los informes "Abril" y "M\&C" se propugnan, merecen especial mención, como pruebas en su contra, las dos siguientes: 
1. Aplicación del tique moderador o copago o gasto por desempeño, con idea de corresponsabilizar a los usuarios, lo que en sí mismo tiene un efecto negativo para la salud al dificultar la accesibilidad a las personas con menor capacidad adquisitiva y más vulnerables. No es justo implantar una tasa por enfermedad $^{7}$, ya que realmente solo afectará a pobres, pensionistas y parados, cuando éstos superan los cuatro millones y mas del $50 \%$ de la población activa son "mileuristas", por lo que, en caso de reducirse la demanda asistencial, lo haría tanto a costa de la innecesaria como de la necesaria, o lo que es igual, de los que tienen más y mayores problemas de salud y menos recursos. Y nos quedamos tan anchos diciendo que el mal uso es siempre un abuso, exigiendo al ciudadano una capacidad de discernimiento a la hora de solicitar asistencia que a veces incluso resulta dificultosa al profesional que los atiende. Por otro lado, debería bastarnos la evidencia de países comunitarios que tienen implantado el tique moderador, también llamado penalizador, como en Alemania, Francia o Bélgica, al tener un gasto sanitario mucho mayor, con peores resultados en salud. No olvidemos que ya existe el copago por medicamentos y que quizá podría hacerse más efectivo ${ }^{8}$ sobre los niveles de renta actuales en diferentes estratos de activos y de pensionistas, si bien el sistema de control (el ajo) podría resultar más caro que lo ahorrado (el pollo).

2. Implantar la autogestión de los centros sanitarios, reforzando la incentivación de los profesionales. Las experiencias que favorecen los excedentes económicos conllevan en general dosis más o menos altas de perversión que repercuten en la cantidad y calidad de las prestaciones asistenciales y con frecuencia en las condiciones laborales de los propios trabajadores de los centros, sin mejorar a cambio las prestaciones o los resultados.

En definitiva, se trata de medidas insolidarias y rechazables que ignoran alevosamente el fracaso cosechado donde ya se implantaron, amén de no contar con la opinión ciudadana que está siendo machaconamente contaminada por quienes esperan cada vez más impacientemente el desmantelamiento del Servicio Sanitario Público Español, empezando por los trozos más apetecibles de la tarta, los económicamente más rentables, en un proceso de difícil retorno, pues como en las guerras deja la "tierra quemada" a su paso.

\section{MÁRGENES SALUDABLES PARA LA MEJORA CONTINUA EN EFICIENCIA}

Sin embargo, y dentro de la alta nota de satisfacción que dan los ciudadanos a su actual sistema sanitario, aún tenemos amplios márgenes "saludables" de mejora, que proponemos, actuando:

- Sobre la cultura de seguridad, de buena praxis y ético-deontológica.

- En el entorno de la medicina basada en la evidencia (MBE).

- Aplicando las recomendaciones de agencias evaluadoras de reconocido prestigio internacional como el National Institute for Health and Clinical Excellence $(\mathrm{NICE})^{9}$, es posible actuar sobre la gestión de lo colectivo, racionalizando la financiación de la tecnología y los medicamentos, de modo que sean costeefectivos; cuestiones difíciles de reconciliar con los intereses particulares del paciente, al que se debe facilitar no todo lo que demande sino todo lo que necesite. Por cuestiones de justicia distributiva debemos tender al ajuste entre las necesidades sociosanitarias y las posibilidades económicas (definidas como objetivos), tema del que el profesional sanitario no está exonerado ni moral ni deontológicamente.

- Introduciendo los "contratos de riesgo compartido", nuevas formas de fijación de precios o de regulación de la financiación de los medicamentos basada en resultados ${ }^{10}$.

- Activando la integración entre niveles asistenciales sanitarios y de éstos con lo social, tendiendo a magnificar la subsidiaridad en lo micro, en lo meso y en lo macro. Son millones los españoles que se re-citan periódicamente y de por vida para revisiones y pruebas complementarias, tanto en las consultas externas del soporte hospitalario, como en el de la $A P$, y de ellos un alto porcentaje sólo porque alguna vez estuvieron enfermos pero curaron o bien se estabilizaron, de modo que deberían ser dados de alta por no enfermedad o bien ser gestionados por su enfermera y médico de familia, dejando a un lado el celo profesional por el que muchos sanos viven con la etiqueta de enfermos.

- Reorientando la gestión del SNS y de los Servicios de Salud autonómicos debidamente cohesionados hacia la prevalente patología crónica.

- Mediante la reconducción progresiva de mutualistas de la función pública (MFP) a la 
cobertura ordinaria, pasando los empleados públicos de ser tratados como colectivo "especial" en lo sanitario a compartir equitativamente la del resto de los ciudadanos, al tiempo que como estrato social con importante capacidad crítica aportara ideas y estabilidad al SNS ${ }^{11}$

- Dando mayor y creíble participación a los propios trabajadores

- Introduciendo innovaciones en gestión de las consultas en favor de la capacidad resolutiva de todos los profesionales sociosanitarios

- Incorporando la cultura desburocratizadora y no medicalizadora de las no enfermedades y del disconfort cotidiano

- Redefiniendo los roles del personal sanitario y no sanitario de Hospitales y Centros de Salud, de modo especial el de las enfermeras, hasta alcanzar su verdadero nivel de competencia en $\mathrm{AP}$, donde además queremos promover el importantísimo papel del personal administrativo para el triaje oportuno en el acceso de los ciudadanos al sistema

- Sobre acuerdos que eviten la variabilidad de actuaciones de los profesionales ante problemas sin solución definida por la comunidad científica o ante las no enfermedades, mediante la correspondiente homogeneización de protocolos y en su caso la aplicación del "primum non nocere" hipocrático, ya que a veces lo mejor es no hacer nada, aunque tenga mala venta

- Haciendo participe a la ciudadanía individual y colectivamente, a través del conocimiento interactivo (información-formación-participación), no solo de "su" problema concreto como pacientes, sino de la situación coetánea del propio Sistema Sanitario para hacer un uso correcto que mantenga saludable al propio Servicio de Salud.

\section{CONCLUSIONES}

Así, como parte de las tan aludidas últimamente "oportunidades de mejora", los puntos citados podrían servirnos aquí y ahora para activar la gestión sanitaria hacia la mejora continua, sobre la base de la justicia distributiva, la que es éticamente demandable, no solo a políticos y gestores, sino a profesionales y ciudadanos. Y ello en función, como reza el artículo 5.1 de la LOPS, del "deber de hacer uso racional de los recursos diagnósticos y terapéuticos, considerando los costes de sus decisiones y evitando su sobre o infrautilización".

Estamos en buen momento, pues.

\section{BIBLIOGRAFÍA}

1. “El Informe Abril". Texto de la Proposición Parlamentaria. 25 de enero de 1990, SerieD, Num. 13. Disponible en: http://www. riberasalud.com/ftp/biblio/07102010131536resumen $\% 20$ informe\%20abril.pdf.

2. Cuenca JA. Informe FEDEA Mckinsey\&Company: Impulsar un cambio posible en el Sistema Sanitario. Med Book, 16 de noviembre de 2009. Disponible en: http://www.medbook.es/ forum/topics/informe-fedea-mckinsey.

3. FADSP. Unas propuestas para el desastre: a propósito del informe sobre sanidad de Mckinsey\&Company. Rev Salud 2000. 2010; 125:13-5.

4. OECD HEALTH DATA 2008. Statistics and Indicators for 30 Countries. Junio de 2008. Disponible en:http://www.oecd.org/document/30/0,3343, en_2649_37407_12968734_1_1_1_37407,00.html.

5. Martín M, Sánchez-Baile M. Nuevas formas de gestión y su impacto sobre las desigualdades. Gac Sanit. 2004; 18(supl 1): 96-101.

6. Leys C. Cómo se privatiza el Servicio Nacional de Salud Británica. Rev Salud 2000. 2007; 113:8-14.

7. Repullo JR. La buena sanidad pública, ¿ni se compra ni se vende?. Rev Salud 2000. 2009; 124:15-20.

8. Gil V, Barrubes J, Alvarez JC, Portilla E. Sostenibilidad financiera del Sistema Sanitario. Informe Antares Consulting, septiembre de 2010.

9. National Institute for Health and Clinical Excellence. 16 de diciembre de 2010. Disponible en: http://www.nice.org.uk/.

10. Puig J, Meneu R. Aplicación de los contratos de riesgo compartido a la financiación de nuevos medicamentos. Rev Gestión Clínica y Sanitaria. 2004; 7: 88-93.

11. Simó J. El techo de cristal de la atención primaria española. Aten Primaria. 2009; 41(10):572-7. 